\title{
Polymorphs of Pentacene and 6,13-Pentacenequinone in Solution and Vapor Phase Growths
}

\author{
Hye Jin Nam, Yoo Jin Kim, ${ }^{\dagger}$ and Duk-Young Jung* \\ Department of Chemistry, Institute of Basic Sciences, Sungkyunkwan Advanced Institute of Nano Technology (SAINT), \\ Sungkyunkwan University, Suwon 440-746, Korea. "E-mail: dyjung@skku.edu \\ ${ }^{\dagger}$ Advanced Industrial Ceramic Team, Korea Institute of Ceramic Engineering and Technology, Icheon 467-843, Korea \\ Received May 4, 2010, Accepted June 30, 2010
}

Key Words: 6,13-Pentacenequinone, Pentacene, Oxidation, Photoluminescence

Conjugated organic compound thin films have received considerable attention because of their great potentials in thinfilm transistors, light emitting diodes and photovoltaic cells. ${ }^{1-4}$ The investigation of organic materials with large charge carrier mobilities has recently become the main research interest in semiconductors. ${ }^{5-7}$ One of the most interesting organic semiconductor materials is pentacene (PEN), which exhibits a remarkably high charge carrier mobility $\left(\geq 1 \mathrm{~cm}^{2} \mathrm{~V}^{-1} \mathrm{~s}^{-1}\right)$, despite being composed of molecular crystals with a relatively weak van der Waals interaction between the molecules. The molecular crystals of polymers, organic and inorganic materials form various polymorphs with different molecular packing structure depending on the preparation conditions such as temperature, pressure, solvent, impurities, supersaturation and carrier-gas flow. The control of the preparation conditions through solutionand vapor-phase growths enables to fabricate crystal structures with different molecular arrangement, which affects the physical, chemical and optical properties. The electronic behavior of molecular crystals also greatly depends on the molecular packing structure, which determines the strength of the intermolecular coupling.

The thin film morphology and chemical impurities also play decisive roles in the charge carrier mobility. Recently, studies identified that the carrier mobility in PEN decreased because of oxygen exposure, where 6,13-pentacenequinone (PQ) received considerable attention as the key compound in the electronic changes that were induced by the mixing ratios of PQ with PEN. ${ }^{8,9}$ Additionally, PQ exhibited a characteristic yellow photoluminescence response, ${ }^{4}$ which indicates that it is an excellent candidate for a UV-light detector. The thin film and single crystal phase structures of PEN have actively been analyzed in several reports, ${ }^{10,11}$ whereas the structural and optical properties of the PQ crystals have rarely been reported, and only one crystal structure has been observed. ${ }^{12}$ In this work, the crystal structure of the PQ crystal exhibited a new polymorph that was obtained in a PEN solution, and also unique photoluminescence property, compared to the vapor-phase PQ films.

PEN (99\%, anhydrous) and PQ (99\%, anhydrous) were purchased from Aldrich Chem. Co. and used without any further purification. The single PEN and PQ crystals were grown through both vacuum sublimation and solution growth in 1,2,4-trichlorobenzene (99\%, anhydrous, Aldrich). For the vapor-phase growth, a pyrex sublimator that was equipped with a heating mantle containing seasand was used as the reactor. Then $30 \mathrm{mg}$ samples of PEN and PQ were placed in each reactor and then the sample containing reactors were heated to $250{ }^{\circ} \mathrm{C}$. The samples were treated at a pressure of about $10^{-2}$ torr for $3 \mathrm{~h}$, and the crystal growth was continued. Thin dark blue PEN plates (PEN-V) and light yellow PQ plates (PQ-V) grew from the wall to the center of the reactors. The solution-phase single PEN and PQ crystals were obtained from the saturated solutions of $\mathrm{PEN}$ in 1,2,4-trichlorobenzene in $\mathrm{N}_{2}$ for PEN and air for PQ at $180^{\circ} \mathrm{C}$. Upon slow cooling to room temperature, orange needlelike PQ crystals (PQ-S) were obtained, whereas only microcrystals were observed for PEN.

The PEN and PQ crystal structures were determined using single crystal X-ray diffraction analyses. Figure 1 shows the microscopic images of the PEN and PQ crystals. The PEN powder that was used as the starting material had a size of $20 \sim 100$ $\mu \mathrm{m}$ and produced dark blue PEN-V crystals that were $1.52 \times$ $0.452 \times 0.008 \mathrm{~mm}^{3}$ after 14 days and $7.03 \times 0.526 \times 0.083 \mathrm{~mm}^{3}$ after 30 days, respectively. PQ-S exhibited orange needle-like crystals that were $2.38 \times 0.180 \times 0.128 \mathrm{~mm}^{3}$, whereas paleyellow needle-like crystals with a size of $7.65 \times 0.700 \times 0.154$ $\mathrm{mm}^{3}$ were obtained for the vapor-grown PQ-V.

The crystal structure of PEN-V had a smaller unit cell volume than the solution-grown product that was previously reported, ${ }^{13}$ whereas the crystal symmetry and the molecular packing structure were the same as the vapor-phase-grown PEN (denoted

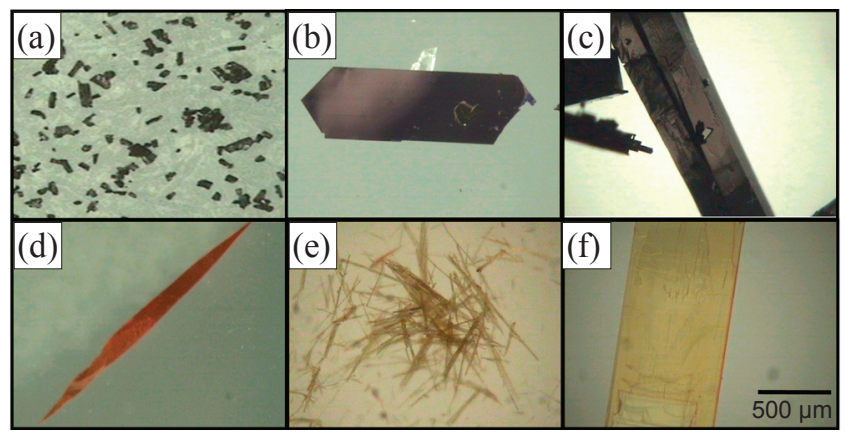

Figure 1. Microscopic images of single crystalline PEN derivatives; (a) commercial PEN chemicals, (b) PEN-V crystal grown on vapor phase (14 days), (c) PEN-V crystal grown on vapor phase (30 days), (d) PQ-S crystal obtained from a saturated solution of PEN, (e) commercial PQ chemicals and (f) PQ-V crystal grown on vapor phase (30 days). 
as Siegrist phase). ${ }^{14}$ The details on the crystal data, intensity collection and refinement are provided in Table S1. The crystal system for PEN-V was triclinic with a $P-1$ symmetry, and the unit cell was $\mathrm{a}=6.2658(10), \mathrm{b}=7.7847(10), \mathrm{c}=14.546(2) \AA$, $\alpha=76.449(10), \beta=87.648(13), \gamma=84.739(12)^{\circ}, d=1.346$ $\mathrm{Mg} / \mathrm{m}^{3}, \mathrm{~V}=686.7(2) \AA^{3}, \mathrm{Z}=2$. In respect to the crystal packing, PEN-V had a face-on-edge herringbone packing structure of planar molecules. PEN-V exhibited a higher molecular packing ratio than the solution-grown PEN, where the van der Waals radii were within the range of 3.6 to $4.0 \AA$, compared to 3.5 to $4.2 \AA$ for the solution-grown PEN.

The crystal structure of PQ-S exhibited a new polymorph different from the PQ crystal (PQ-R) that was previously reported. ${ }^{12}$ The crystal system for the PQ-S was monoclinic with a $P 2_{1} / \mathrm{c}$ symmetry and the unit cell was $\mathrm{a}=3.9580(8), \mathrm{b}=$ 9.4760(19), $\mathrm{c}=18.813(4) \AA, \alpha=90, \beta=95.635(4), \gamma=90^{\circ}, d=$ $1.458 \mathrm{Mg} / \mathrm{m}^{3}, \mathrm{~V}=702.2(2) \AA^{3}, \mathrm{Z}=2$. The volume of the unit cell was $2.2 \%$ smaller than PQ-R, resulting in a closer molecular packing. The molecular packing of the present $P Q-V$ exhibited a very similar structure to the previously reported $\mathrm{PQ}$ data. ${ }^{12,14}$ The crystal system was monoclinic with a $P 2_{1} / \mathrm{c}$ symmetry and the unit cell was $\mathrm{a}=4.9455(6), \mathrm{b}=8.1654(9), \mathrm{c}=17.772(2) \AA$, $\alpha=90, \beta=93.221(8), \gamma=90^{\circ}, d=1.429 \mathrm{Mg} / \mathrm{m}^{3}, \mathrm{~V}=716.73(14)$ $\AA^{3}, Z=2$. Both the PQ-S and PQ-V crystals exhibited a zigzag structure of molecules that were arranged with a coplanar stacking (See supporting data.). In the PQ-S crystal structure, the intermolecular $\mathrm{O} \cdots \mathrm{H}$ distances were in the range from 2.7 to $3.3 \AA$, which was shorter than PQ-R $(2.6 \sim 4.6 \AA)$. Interestingly, for PQ-V, all of the $\mathrm{O} \cdots \mathrm{H}$ bond distances were the same with a value of $2.7 \AA$. The intermolecular carbon-carbon contacts along the $c$-axis were within the range of 3.6 to $4.3 \AA$, whereas the contact distances of 9.6 to $11 \AA$ along the $a$-axis were shorter, compared to PQ-V and PQ-R (11 $13 \AA$ ). PQ-S demonstrated a closer molecular packing with smaller interlayer angles between the molecule layers, as well as the intermolecular carbon-carbon contacts. PQ-S exhibited much smaller interlayer angles $\left(\alpha=\beta=85^{\circ}\right)$ than PQ-V or PQ-R $\left(\alpha=\beta=106^{\circ}\right)$ (See supporting data).

The X-ray diffraction data were collected for the crystal samples in order to determine the growth direction. Figure 2 shows

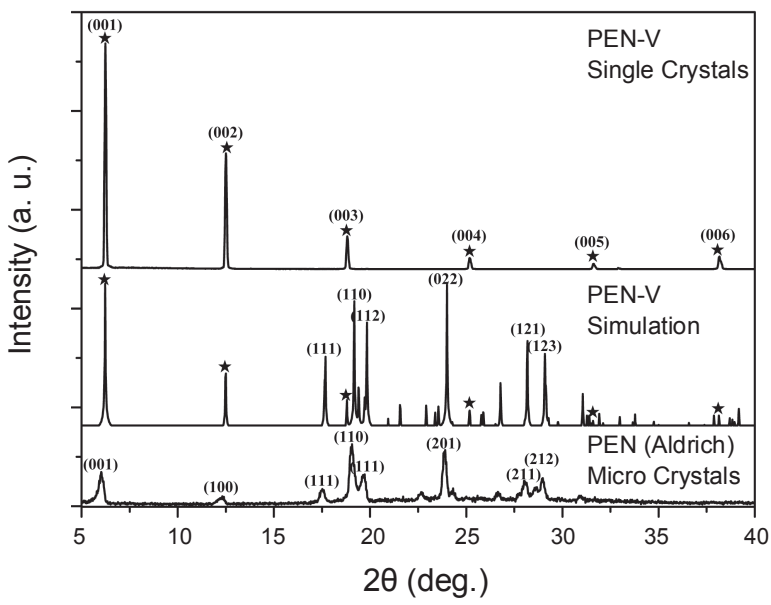

Figure 2. XRD data of PEN-V crystal, simulation obtained from single crystal data and commercial PEN micro crystals. that the PEN-V data fit well with the simulation data based on the single crystal analysis, with a highly preferred orientation compared to the commercial PEN at the six $(00 l)(l=1,2,3, \cdots)$ peaks with a basal spacing of $14.1 \AA^{14}$ The simulation data provided the supplementary peaks that were indexed to the (111), (110), (112), (022), (121) and (123) planes. The peaks for the commercial PEN corresponded to the bulk crystal with different $2 \theta$ values from PEN-V. ${ }^{13}$

The PQ crystals also exhibited preferred orientations at the $(00 l)(l=2,4,6, \cdots)$ planes and had $d$-spacings of 9.4 and $8.9 \AA$ for PQ-S and PQ-V, respectively, compared to $8.7 \AA$ for PQ-R (See supporting data). The $d$-spacing value of the PQ-R showed very good agreement with the value of the vacuum-deposited thin film PQ. ${ }^{4}$ The simulation data for PQ provided the peaks for the $(011),(012),(112)$ and (104) planes as well as $(00 l)$, which corresponded to the PQ-R data (JCPDS No. 47-2123). Notably, the peak positions of PQ-S differed from PQ-V or PQ-R, and the diffraction peaks with a preferred orientation were observed at $2 \theta=9.38,10.4,18.8$ and $28.4^{\circ}$, corresponding to the $(002),(011),(004)$ and $(006)$ planes, respectively. The diffraction peaks for PQ-S show that PEN oxidized to PQ with a new polymorph during the solution-phase growth.

Figure 4 shows the normalized photoluminescence emission spectra of PQ-S and PQ-V. PQ-V exhibited two characteristic luminescence peaks, corresponding to the typical PL spectra of the 6,13-disubstituted PEN derivatives, but only one broad peak was observed for PQ-S. In the emission spectra, the most intense luminescence peaks were observed in the yellow regions at $556 \mathrm{~nm}$ for PQ-V and $586 \mathrm{~nm}$ for PQ-S without quenching that often occurrs in organic crystal systems ${ }^{15}$ and shifted to a shorter wavelength region for PQ-V and a longer wavelength region for $\mathrm{PQ}-\mathrm{S}$ compared to the thin film phase $\mathrm{PQ}{ }^{4,16}$

In summary, a new polymorph of PQ-S was demonstrated for the first time. This polymorph was grown in solution through the oxidation of PEN, creating a zigzag structure that was tilted by $21^{\circ}$ with respected to $a$-axis. The PQ-V crystal had a packing structure similar to a thin-film phase, where oxygen was a critical factor in determining the molecular packing. The remarkable shift in the PL maximum wavelength between PQ-S and $\mathrm{PQ}-\mathrm{V}$ of up to $30 \mathrm{~nm}$ confirmed that the molecular packing

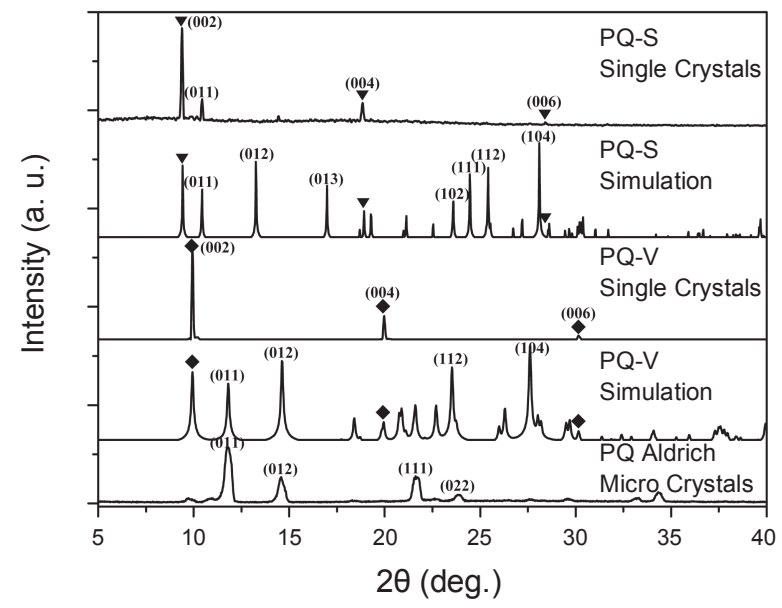

Figure 3. Experimental and simulated $X R D$ data of $P Q-V$ and $P Q-S$. 


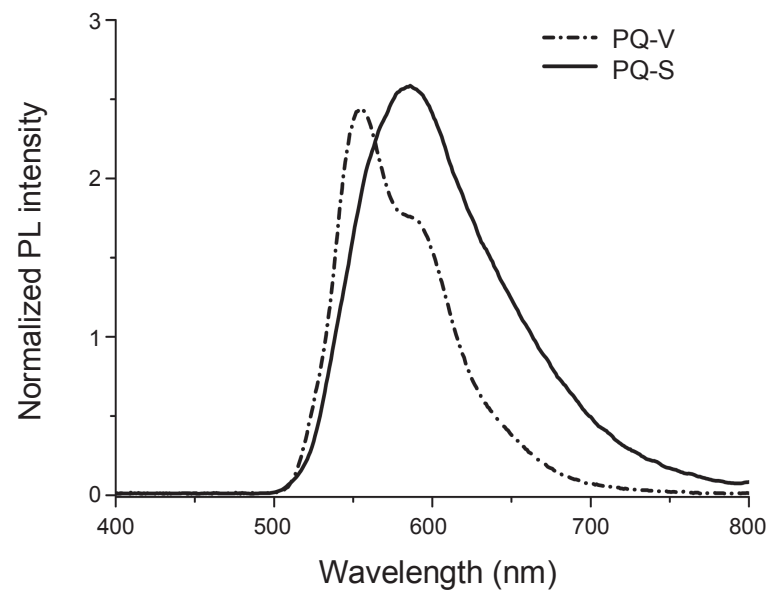

Figure 4. Normalized photoluminescence emission spectra of PQ-S and PQ-V on glass substrate.

difference strongly influenced the light emitting properties. The structural changes from pentacene to pentacenequinone that occured through oxidation were carefully studied in the single crystal analysis and provided useful information on the quality and performance of the pentacene-containing devices. This present study on the polymorph of pentacene and pentacenequinone provides some basic structural data.

\section{Experimental Section}

Characterization. The X-ray data were collected using a Bruker Smart APEX2 diffractometer that was equipped with a Mo X-ray tube. The absorption corrections were made using SADABS based upon the Laue symmetry of the equivalent reflections. All of the calculations were carried out with SHELXTL programs using direct methods. The XRD patterns were measured using a Rigaku X-ray diffractometer, D/MAX-2000 Ultima, with a $\theta \sim 2 \theta$ scanning mode. The measurements were performed at $40 \mathrm{kV}, 30 \mathrm{~mA}$, and $\mathrm{Cu} \mathrm{K} \alpha(\lambda=1.5405 \AA)$. The XRD peak positions of the films were calibrated using $\mathrm{Si}(100)$ $\left(2 \theta=32.9^{\circ}\right)$ as the sample holder. The photoluminescence measurements were carried out at room temperature using a light source consisting of a $500 \mathrm{~W} \mathrm{Hg}(\mathrm{Xe})$-Arc lamp and a monochromator from 350 to $800 \mathrm{~nm}$.

Acknowledgments. This research was supported from the Korea Research Foundation Grant funded by Korean government (MOEHRD) (KRF-2008-005-J00702), the National Re- search Laboratory Program of Korea Science and Engineering Foundation (Program No. ROA-2007-000-10020-0), the Research Center of Break-through Technology Program through the Korea Institute of Energy Technology Evaluation and Planning (KETEP) (Program No. 2009-3021010030).

Supplementary Material Available. Crystallographic data for the structure reported here have been deposited with the Cambridge Crystallographic Data Centre (Deposition No. CCDC786150). These data can be obtained free of charge at www. ccdc.cam.ac.uk/conts/retrieving.html [or from the Cambridge Crystallographic Data Centre (CCDC), 12 Union Road, Cambridge CB2 1EZ, UK; fax: +44(0) 1222-336033; email: deposit @ccdc.cam.ac.uk]. Supplementary crystallographic data and crystal packing structures for PEN-V, PQ-S and PQ-V crystals are available.

\section{References}

1. Tang, C. W.; Van Slyke, S. A. Appl. Phys. Lett. 1987, 51, 913.

2. Burroughes, J. H.; Bradley, D. D. C.; Brown, A. R.; Marks, R. N.; Mackay, K.; Friend, R. H.; Burn, P. L.; Holmes, A. B. Nature 1990, 347,539

3. Gundlach, D. J.; Lin, Y. Y.; Jackson, T. N.; Nelson, S. F.; Schlom, D. G. IEEE Electron Device Lett. 1997, 18, 87.

4. Lee, J. Y.; Kim, S. S.; Kim, K. B.; Kim, J. H.; Im, S. Appl. Phys. Lett. 2004, 84, 1701

5. Peumans, P.; Yakimov, A.; Forrest, S. R. J. Appl. Phys. 2003, 93, 3693.

6. Salzman, R. F.; Xue, J.; Rand, B. P.; Alexander, A.; Thompson, M. E.; Forrest, S. R. Org. Electron. 2005, 6, 242.

7. Payne, M. M.; Parkin, S. R.; Anthony, J. E.; Kuo, C. C.; Jackson, T. N. J. Am. Chem. Soc. 2005, 127, 4986.

8. Jurchescu, O. D.; Baas, J.; Palstra, T. T. M. Appl. Phys. Lett. 2004, $84,3061$.

9. Gomar-Nadal, E.; Conrad, B. R.; Cullen, W. G.; Willams, E. D. J. Phys. Chem. C 2008, 112, 5646.

10. Yoshida, H.; Sato, N. Phys. Rev. B 2008, 77, 235205.

11. Kafer, D.; Helou, M. E.; Gemel, C.; Witte, G. Crystal Growth \& Design 2008, 8, 3053.

12. Dzyabchenko, A. V.; Zavodnik, V. E.; Belsky, V. K. Acta Crystallogr. B 1979, 35, 2250.

13. Campbell, R. B.; Robertson, J. M.; Trotter, J. Acta Crystallogr. 1962, 15, 289

14. Siegrist, T.; Kloc, C.; Schon, J. H.; Batlogg, B.; Haddon, R. C.; Berg, S.; Thomas, G. A. Angew. Chem., Int. Ed. 2001, 40, 1732.

15. Pope, M.; Swenberg, C. E. Electronic Processes in Organic Crystals and Polymers, 2nd ed.; Oxford University Press: New York, 1999; pp 40-43.

16. Parisse, P.; Luciani, D.; Santucci1, S.; Zuppella, P.; Tucceri, P.; Reale, A.; Ottaviano, L. J. Phys. D: Appl. Phys. 2008, 41, 112003. 